\title{
CHARACTERIZATIONS OF HIGHER DERIVATIONS AND JORDAN HIGHER DERIVATIONS ON CSL ALGEBRAS
}

\author{
JIANKUI LI $^{\bowtie}$ and JIANBIN GUO
}

(Received 8 September 2010)

\begin{abstract}
Let $\mathcal{L}$ be a commutative subspace lattice and $\mathcal{A}=\operatorname{Alg} \mathcal{L}$. It is shown that every Jordan higher derivation from $\mathcal{A}$ into itself is a higher derivation. We say that $D=\left(\delta_{i}\right)_{i \in \mathbb{N}}$ is a higher derivable linear mapping at $G$ if $\delta_{n}(A B)=\sum_{i+j=n} \delta_{i}(A) \delta_{j}(B)$ for all $n \in \mathbb{N}$ and $A, B \in \mathcal{A}$ with $A B=G$. We also prove that if $D=\left(\delta_{i}\right)_{i \in \mathbb{N}}$ is a bounded higher derivable linear mapping at 0 from $\mathcal{A}$ into itself and $\delta_{n}(I)=0$ for all $n \geq 1$, or $D=\left(\delta_{i}\right)_{i \in \mathbb{N}}$ is a higher derivable linear mapping at $I$ from $\mathcal{A}$ into itself, then $D=\left(\delta_{i}\right)_{i \in \mathbb{N}}$ is a higher derivation.
\end{abstract}

2010 Mathematics subject classification: primary 47B47; secondary 47L35.

Keywords and phrases: CSL algebra, higher derivable linear mapping, higher derivation, Jordan higher derivation.

\section{Introduction}

Let $H$ be a separable complex Hilbert space and let $B(H)$ be the set of all bounded linear operators on $H$. By a subspace lattice on $H$, we mean a collection $\mathcal{L}$ of closed subspaces of $H$ with 0 and $H$ in $\mathcal{L}$ such that for every family $\left\{M_{r}\right\}$ of elements of $\mathcal{L}$, both $\cap M_{r}$ and $\vee M_{r}$ belong to $\mathcal{L}$. For a subspace lattice $\mathcal{L}$ of $H$, let $\operatorname{Alg} \mathcal{L}$ denote the algebra of all operators in $B(H)$ that leave members of $\mathcal{L}$ invariant; and for a subalgebra $\mathcal{A}$ of $B(H)$, let Lat $\mathcal{A}$ denote the lattice of all closed subspaces of $H$ that are invariant under all operators in $\mathcal{A}$. We disregard the distinction between a subspace and the orthogonal projection onto it. It is not difficult to show that $\operatorname{Alg} \mathcal{L}$ is closed in the weak operator topology, and is a unital Banach algebra. An algebra $\mathcal{A}$ is called reflexive if $\operatorname{Alg} \operatorname{Lat} \mathcal{A}=\mathcal{A}$; and dually, a subspace lattice is called reflexive if Lat $\operatorname{Alg} \mathcal{L}=\mathcal{L}$. Every reflexive algebra is of the form $\operatorname{Alg} \mathcal{L}$ for some subspace lattice $\mathcal{L}$ and every reflexive lattice is of the form Lat $\mathcal{A}$ for some weakly-closed unital algebra $\mathcal{A}$. A subspace lattice $\mathcal{L}$ on a Hilbert space $H$ is called a commutative subspace lattice (CSL), if all projections in $\mathcal{L}$ commute pairwise. If $\mathcal{L}$ is a CSL, then $\operatorname{Alg} \mathcal{L}$ is called a CSL algebra. By [5], we know that if $\mathcal{L}$ is a CSL, then $\mathcal{L}$ is reflexive.

This work is supported by NSF of China.

(C) 2011 Australian Mathematical Publishing Association Inc. 0004-9727/2011 \$16.00 
Let $\mathcal{R}$ be a commutative ring with identity and $\mathcal{A}$ be a unital associative algebra over $\mathcal{R}$. Let $D=\left(\delta_{i}\right)_{i \in \mathbb{N}}$ be a family of linear mappings on $\mathcal{A}$ such that $\delta_{0}=\operatorname{id}_{\mathcal{A}}$. $D$ is called a higher derivation if $\delta_{n}(x y)=\sum_{i+j=n} \delta_{i}(x) \delta_{j}(y)$ for all $n \in \mathbb{N}$ and $x, y \in \mathcal{A}$; $D$ is called a Jordan higher derivation if $\delta_{n}\left(x^{2}\right)=\sum_{i+j=n} \delta_{i}(x) \delta_{j}(x)$ for all $n \in \mathbb{N}$ and $x \in \mathcal{A}$. Note that $\delta_{1}$ is a Jordan derivation if $D=\left(\delta_{i}\right)_{i \in \mathbb{N}}$ is a Jordan higher derivation. It is well known that every derivation is a Jordan derivation and that the converse in general is not true. In [7], Herstein showed that every Jordan derivation from a 2-torsion-free prime ring into itself is a derivation. In [2], Brešar generalized Herstein's result to 2-torsion-free semiprime rings. Likewise, every higher derivation is a Jordan higher derivation and the converse in general is not true. In [6], Ferrero and Haetinger generalized Brešar's result to the Jordan higher derivations, showing that every Jordan higher derivation of a 2-torsion-free semiprime ring is a higher derivation. For other results, see [14, 15]. In [13], Lu proved that each Jordan derivation from a CSL algebra into itself is a derivation. In Section 2, we generalize Lu's result to the case of Jordan higher derivations.

In general, there are two directions in the study of the local actions of derivations of operator algebras. One is the local derivation problem (for example, see $[4,9,10]$ ). The other is to examine the conditions under which derivations of operator algebras can be completely determined by the action on some sets of operators (for example, see $[1,3,8,11])$. In Section 3 we study the local actions of higher derivations. We show that if the family of bounded linear mappings $D=\left(\delta_{i}\right)_{i \in \mathbb{N}}$ on a CSL algebra $\mathcal{A}$ satisfies $\sum_{i+j=n} \delta_{i}(A) \delta_{j}(B)=0$ for all $n \in \mathbb{N}$ and $A, B \in \mathcal{A}$ with $A B=0$ and $\delta_{n}(I)=0$ for all $n \geq 1$, then $D=\left(\delta_{i}\right)_{i \in \mathbb{N}}$ is a higher derivation. We also prove that if $\sum_{i+j=n} \delta_{i}(A) \delta_{j}(B)=0$ for all $n \geq 1$ and $A, B \in \mathcal{A}$ with $A B=I$, then $D=\left(\delta_{i}\right)_{i \in \mathbb{N}}$ is a Jordan higher derivation.

\section{Jordan higher derivations on CSL algebras}

The following lemma, due to Ferrero and Haetinger [6], will be used repeatedly.

LEMMA 2.1. Let $\mathcal{A}$ be an algebra over a 2-torsion-free commutative ring and $D=$ $\left(\delta_{i}\right)_{i \in \mathbb{N}}$ be a Jordan higher derivation from $\mathcal{A}$ into itself. Then for all $x, y \in \mathcal{A}$ and each $n \in \mathbb{N}$ :

(a) $\delta_{n}(x y+y x)=\sum_{i+j=n}\left(\delta_{i}(x) \delta_{j}(y)+\delta_{i}(y) \delta_{j}(x)\right)$;

(b) $\quad \delta_{n}(x y x)=\sum_{i+j+k=n} \delta_{i}(x) \delta_{j}(y) \delta_{k}(x)$.

To show one of our main results, we need the following lemmas.

LEMMA 2.2. Let $\mathcal{L}$ be a CSL on $H$ and $D=\left(\delta_{i}\right)_{i \in \mathbb{N}}$ be a Jordan higher derivation from $\operatorname{Alg} \mathcal{L}$ into itself. Then for any $E \in \mathcal{L}$ and $n \geq 1$ :

(a) $\delta_{n}(E)=E \delta_{n}(E)(I-E)$;

(b) $\delta_{n}(I-E)=E \delta_{n}(I-E)(I-E)$.

PROOF. When $n=1$, it is easy to show that $\delta(E)=E \delta(E)(I-E)$. Now we assume that $\delta_{m}(E)=E \delta_{m}(E)(I-E)$ for all $1 \leq m<n$. According to the definition of Jordan 
higher derivation,

$$
\delta_{n}(E)=E \delta_{n}(E)+\delta_{n}(E) E+\sum_{\substack{i+j=n \\ i, j \geq 1}} \delta_{i}(E) \delta_{j}(E) .
$$

By assumption $\delta_{n}(E)=E \delta_{n}(E)+\delta_{n}(E) E$ and so $E \delta_{n}(E) E=(I-E) \delta_{n}(E)$ $(I-E)=0$. This means that

$$
\delta_{n}(E)=E \delta_{n}(E)(I-E)
$$

for all $n \geq 1$ and (a) is proved. By (a), $\delta_{n}(I)=0$ for all $n \geq 1$. Thus we have

$$
\delta_{n}(E)=-\delta_{n}(I-E)
$$

and

$$
\delta_{n}(I-E)=-E \delta_{n}(E)(I-E)=E \delta_{n}(I-E)(I-E)
$$

for all $n \geq 1$. This proves (b).

Lemma 2.3. Let $\mathcal{L}$ and $D$ be as in Lemma 2.2. Then, for all $n \in \mathbb{N}, A \in \operatorname{Alg} \mathcal{L}$ and $E \in \mathcal{L}:$

(a) $\delta_{n}(A E)=\sum_{i+j=n} \delta_{i}(A) \delta_{j}(E)$;

(b) $\delta_{n}(A(I-E))=\sum_{i+j=n} \delta_{i}(A) \delta_{j}(I-E)$;

(c) $\delta_{n}(E A)=\sum_{i+j=n} \delta_{i}(E) \delta_{j}(A)$;

(d) $\delta_{n}((I-E) A)=\sum_{i+j=n} \delta_{i}(I-E) \delta_{j}(A)$.

PROOF. We prove only (a) and (b). The proofs of (c) and (d) are similar.

By Lemmas 2.1 and 2.2,

$$
\begin{aligned}
\delta_{n}(A E) & =\delta_{n}(E A E)=\sum_{i+j+k=n} \delta_{i}(E) \delta_{j}(A) \delta_{k}(E) \\
& =\sum_{\substack{i+j+k=n \\
i \geq 1}} \delta_{i}(E) \delta_{j}(A) \delta_{k}(E)+\sum_{i+j=n} E \delta_{i}(A) \delta_{j}(E) \\
& =\sum_{i+j=n} \delta_{i}(A) \delta_{j}(E)
\end{aligned}
$$

and

$$
\begin{aligned}
\delta_{n}(A(I-E)) & =\delta_{n}(A)-\delta_{n}(A E)=\delta_{n}(A)-\sum_{i+j=n} \delta_{i}(A) \delta_{j}(E) \\
& =\delta_{n}(A)(I-E)+\sum_{\substack{i+j=n \\
j \geq 1}} \delta_{i}(A) \delta_{j}(I-E) \\
& =\sum_{i+j=n} \delta_{i}(A) \delta_{j}(I-E) .
\end{aligned}
$$

This concludes the proof. 
LEMMA 2.4. Let $\mathcal{L}$ and $D$ be as in Lemma 2.2. Then for all $n \in \mathbb{N}, S, T \in \operatorname{Alg} \mathcal{L}$ and $E \in \mathcal{L}:$

(a) $\delta_{n}(S E T(I-E))=\sum_{i+j=n} \delta_{i}(S) \delta_{j}(E T(I-E))$;

(b) $\delta_{n}(E S(I-E) T)=\sum_{i+j=n} \delta_{i}(E S(I-E)) \delta_{j}(T)$.

Proof. By Lemma 2.1, for every $T \in \operatorname{Alg} \mathcal{L}$ and $E \in \mathcal{L}$,

$$
\begin{aligned}
\delta_{n}(E T(I-E))= & \delta_{n}(E E T(I-E)+E T(I-E) E) \\
= & \sum_{i+j=n}\left(\delta_{i}(E) \delta_{j}(E T(I-E))+\delta_{i}(E T(I-E)) \delta_{j}(E)\right) \\
= & E \delta_{n}(E T(I-E))+\sum_{\substack{i+j=n \\
i \geq 1}} \delta_{i}(E) \delta_{j}(E T(I-E)) \\
& \quad+\delta_{n}(E T(I-E)) E+\sum_{\substack{i+j=n \\
j \geq 1}} \delta_{i}(E T(I-E)) \delta_{j}(E) .
\end{aligned}
$$

By Lemma 2.2, we know that, for any $E \in \mathcal{L}$ and $n \geq 1, \delta_{n}(E)=E \delta_{n}(E)(I-E)$ and $\delta_{n}(I-E)=E \delta_{n}(I-E)(I-E)$. Thus by the equations above, we obtain

$$
E \delta_{n}(E T(I-E)) E=(I-E) \delta_{n}(E T(I-E))(I-E)=0 .
$$

This implies that

$$
\delta_{n}(E T(I-E))=E \delta_{n}(E T(I-E))(I-E) .
$$

By Lemma 2.3, we can easily obtain

$$
(I-E) \delta_{n}(E T E)=0 \quad \text { and } \quad \delta_{n}((I-E) T(I-E)) E=0 .
$$

Hence for $S, T \in \operatorname{Alg} \mathcal{L}$ and $E \in \mathcal{L}$,

$$
\begin{aligned}
\delta_{n}(S E T(I-E)) & =\delta_{n}(E S E E T(I-E)+E T(I-E) E S E) \\
& =\sum_{i+j=n}\left(\delta_{i}(E S E) \delta_{j}(E T(I-E))+\delta_{i}(E T(I-E)) \delta_{j}(E S E)\right) \\
& =\sum_{i+j=n} \delta_{i}(E S E) \delta_{j}(E T(I-E)) \\
& =\sum_{i+j=n} \sum_{e+f+g=i} \delta_{e}(E) \delta_{f}(S) \delta_{g}(E) \delta_{j}(E T(I-E)) \\
& =\sum_{i+j=n} E \delta_{i}(S) E \delta_{j}(E T(I-E)) \\
& =\sum_{i+j=n} \delta_{i}(S) \delta_{j}(E T(I-E)) .
\end{aligned}
$$


Similarly, for all $S, T \in \operatorname{Alg} \mathcal{L}$ and $E \in \mathcal{L}$,

$$
\delta_{n}(E S(I-E) T)=\sum_{i+j=n} \delta_{i}(E S(I-E)) \delta_{j}(T)
$$

This concludes the proof.

Let $\mathcal{L}^{\perp}$ be the lattice $\{I-E: E \in \mathcal{L}\}$ and $\mathcal{L}^{\prime}$ be the commutant of $\mathcal{L}$. It is easy to verify that $(\operatorname{Alg} \mathcal{L})^{*}=\operatorname{Alg} \mathcal{L}^{\perp}$ for any lattice $\mathcal{L}$ on $H$ and the diagonal $(\operatorname{Alg} \mathcal{L}) \cap(\operatorname{Alg} \mathcal{L})^{*}=\mathcal{L}^{\prime}$ is a von Neumann algebra. Given a CSL $\mathcal{L}$ on a Hilbert space $H$, we define $G_{1}(\mathcal{L})$ and $G_{2}(\mathcal{L})$ to be the projections onto the closures of the linear spans of

$$
\{E A(I-E) x: E \in \mathcal{L}, A \in \operatorname{Alg} \mathcal{L}, x \in H\}
$$

and

$$
\left\{(I-E) A^{*} E x: E \in \mathcal{L}, A \in \operatorname{Alg} \mathcal{L}, x \in H\right\}
$$

respectively. For simplicity, we write $G_{1}$ and $G_{2}$ for $G_{1}(\mathcal{L})$ and $G_{2}(\mathcal{L})$. Since the CSL is reflexive, it is easy to verify that $G_{1} \in \mathcal{L}$ and $G_{2} \in \mathcal{L}^{\perp}$. In [13], Lu showed that $G_{1} \vee G_{2} \in \mathcal{L} \cap \mathcal{L}^{\perp}$ and $(\operatorname{Alg} \mathcal{L})\left(I-G_{1} \vee G_{2}\right) \subseteq \mathcal{L}^{\prime}$

THEOREM 2.5. Let $\mathcal{L}$ be a CSL on $H$. If $D=\left(\delta_{i}\right)_{i \in \mathbb{N}}$ is a Jordan higher derivation from $\operatorname{Alg} \mathcal{L}$ into itself, then $D=\left(\delta_{i}\right)_{i \in \mathbb{N}}$ is a higher derivation.

PROOF. We divide the proof into two cases.

Case 1. Suppose that $G_{1} \vee G_{2}=I$.

Let $D=\left(\delta_{i}\right)_{i \in \mathbb{N}}$ be a Jordan higher derivation from $\operatorname{Alg} \mathcal{L}$ into itself. Then $\delta_{1}$ is a Jordan derivation, so by [13, Theorem 3.2], we immediately obtain $\delta_{1}(A B)=$ $\delta_{1}(A) B+A \delta_{1}(B)$ for all $A, B \in \operatorname{Alg} \mathcal{L}$. Now we assume that

$$
\delta_{m}(A B)=\sum_{i+j=m} \delta_{i}(A) \delta_{j}(B)
$$

for all $A, B \in \operatorname{Alg} \mathcal{L}$ and for all $1 \leq m<n$.

Let $A, B \in \operatorname{Alg} \mathcal{L}$. Then by Lemma 2.4, for $T \in \operatorname{Alg} \mathcal{L}$ and $E \in \mathcal{L}$,

$$
\begin{aligned}
\delta_{n}(A B E T(I-E)) & =\sum_{i+j=n} \delta_{i}(A B) \delta_{j}(E T(I-E)) \\
& =\delta_{n}(A B) E T(I-E)+\sum_{\substack{i+j=n \\
j \geq 1}} \delta_{i}(A B) \delta_{j}(E T(I-E)),
\end{aligned}
$$




$$
\begin{aligned}
\delta_{n}(A B E T(I-E))= & \sum_{i+j=n} \delta_{i}(A) \delta_{j}(B E T(I-E)) \\
= & \sum_{i+j+k=n} \delta_{i}(A) \delta_{j}(B) \delta_{k}(E T(I-E)) \\
= & \sum_{i+j=n} \delta_{i}(A) \delta_{j}(B) E T(I-E) \\
& \quad+\sum_{\substack{i+j+k=n \\
k \geq 1}} \delta_{i}(A) \delta_{j}(B) \delta_{k}(E T(I-E)) .
\end{aligned}
$$

Combining (2.1) and (2.2) and using the induction hypothesis, we arrive at

$$
\left(\delta_{n}(A B)-\sum_{i+j=n} \delta_{i}(A) \delta_{j}(B)\right) E T(I-E)=0 .
$$

Hence by the arbitrariness of $E$ and $T$, we obtain

$$
\left(\delta_{n}(A B)-\sum_{i+j=n} \delta_{i}(A) \delta_{j}(B)\right) G_{1}=0 .
$$

Define $\delta_{n}^{*}(A)=\left(\delta_{n}\left(A^{*}\right)\right)^{*}$ for all $n \in \mathbb{N}$ and any $A \in \operatorname{Alg} \mathcal{L}^{\perp}$. Let $D^{*}=\left(\delta_{i}^{*}\right)_{i \in \mathbb{N}}$. If $A$ and $B$ belong to $\operatorname{Alg} \mathcal{L}^{\perp}$, then

$$
\delta_{n}^{*}\left(A^{2}\right)=\left(\delta_{n}\left(\left(A^{*}\right)^{2}\right)\right)^{*}=\sum_{i+j=n}\left(\delta_{i}\left(A^{*}\right)\right)^{*}\left(\delta_{j}\left(A^{*}\right)\right)^{*}=\sum_{i+j=n} \delta_{i}^{*}(A) \delta_{j}^{*}(A) .
$$

So $D^{*}=\left(\delta_{i}^{*}\right)_{i \in \mathbb{N}}$ is a Jordan higher derivation on $\operatorname{Alg} \mathcal{L}^{\perp}$. With the proof similar to the proof of (2.3), we have, for all $A, B \in \operatorname{Alg} \mathcal{L}$,

$$
G_{2}\left(\delta_{n}(A B)-\sum_{i+j=n} \delta_{i}(A) \delta_{j}(B)\right)=0 .
$$

Since $G_{1} \in \mathcal{L}$, by Lemma 2.3, we have, for all $A, B \in \operatorname{Alg} \mathcal{L}$,

$$
\begin{aligned}
& \left(\delta_{n}\left(A B G_{1}\right)-\sum_{i+j=n} \delta_{i}(A) \delta_{j}\left(B G_{1}\right)\right)\left(I-G_{1}\right) \\
& =\left(\sum_{i+j=n} \delta_{i}(A B) \delta_{j}\left(G_{1}\right)-\sum_{e+f+j=n} \delta_{e}(A) \delta_{f}(B) \delta_{j}\left(G_{1}\right)\right)\left(I-G_{1}\right) \\
& =\sum_{i+j=n}\left(\delta_{i}(A B)-\sum_{e+f=i} \delta_{e}(A) \delta_{f}(B)\right) \delta_{j}\left(G_{1}\right)\left(I-G_{1}\right) \\
& =\sum_{i+j=n}\left(\delta_{i}(A B)-\sum_{e+f=i} \delta_{e}(A) \delta_{f}(B)\right) \delta_{j}\left(G_{1}\right)\left(I-G_{1}\right)
\end{aligned}
$$


and

$$
\begin{aligned}
G_{1}( & \left.\delta_{n}\left(\left(I-G_{1}\right) A B\right)-\sum_{i+j=n} \delta_{i}\left(\left(I-G_{1}\right) A\right) \delta_{j}(B)\right) \\
= & G_{1}\left(\sum_{i+j=n} \delta_{i}\left(I-G_{1}\right) \delta_{j}(A B)-\sum_{i+e+f=n} \delta_{i}\left(I-G_{1}\right) \delta_{e}(A) \delta_{f}(B)\right) \\
= & G_{1} \sum_{i+j=n} \delta_{i}\left(I-G_{1}\right)\left(\delta_{j}(A B)-\sum_{e+f=j} \delta_{e}(A) \delta_{f}(B)\right) \\
= & G_{1} \sum_{i+j=n} \delta_{i}\left(I-G_{1}\right)\left(\delta_{j}(A B)-\sum_{i \geq 1} \delta_{e}(A) \delta_{f}(B)\right) .
\end{aligned}
$$

By the assumption,

$$
\left(\delta_{n}\left(A B G_{1}\right)-\sum_{i+j=n} \delta_{i}(A) \delta_{j}\left(B G_{1}\right)\right)\left(I-G_{1}\right)=0
$$

and

$$
G_{1}\left(\delta_{n}\left(\left(I-G_{1}\right) A B\right)-\sum_{i+j=n} \delta_{i}\left(\left(I-G_{1}\right) A\right) \delta_{j}(B)\right)=0 .
$$

So by (2.3)-(2.6) and $G_{1} \vee G_{2}=I$, we obtain

$$
\delta_{n}\left(A B G_{1}\right)=\sum_{i+j=n} \delta_{i}(A) \delta_{j}\left(B G_{1}\right)
$$

and

$$
\delta_{n}\left(\left(I-G_{1}\right) A B\right)=\sum_{i+j=n} \delta_{i}\left(\left(I-G_{1}\right) A\right) \delta_{j}(B)
$$

for all $A, B \in \operatorname{Alg} \mathcal{L}$.

Note that by Lemma 2.4, we also have

$$
\delta_{n}\left(A G_{1} B\left(I-G_{1}\right)\right)=\sum_{i+j=n} \delta_{i}(A) \delta_{j}\left(G_{1} B\left(I-G_{1}\right)\right)
$$

and

$$
\delta_{n}\left(G_{1} A\left(I-G_{1}\right) B\right)=\sum_{i+j=n} \delta_{i}\left(G_{1} A\left(I-G_{1}\right)\right) \delta_{j}(B)
$$

for all $A, B \in \operatorname{Alg} \mathcal{L}$. 
Therefore by (2.7)-(2.10) and Lemma 2.3,

$$
\begin{aligned}
\delta_{n}(A B)= & \delta_{n}\left(A B G_{1}+A G_{1} B\left(I-G_{1}\right)+G_{1} A\left(I-G_{1}\right) B+\left(I-G_{1}\right) A B\right) \\
= & \sum_{i+j=n} \delta_{i}(A) \delta_{j}\left(B G_{1}\right)+\sum_{i+j=n} \delta_{i}(A) \delta_{j}\left(G_{1} B\left(I-G_{1}\right)\right) \\
& \quad+\sum_{i+j=n} \delta_{i}\left(G_{1} A\left(I-G_{1}\right)\right) \delta_{j}(B)+\sum_{i+j=n} \delta_{i}\left(\left(I-G_{1}\right) A\right) \delta_{j}(B) \\
= & \sum_{i+j=n} \delta_{i}(A) \delta_{j}\left(G_{1} B\right)+\sum_{i+j=n} \delta_{i}\left(A\left(I-G_{1}\right)\right) \delta_{j}(B) \\
= & \sum_{i+j+k=n} \delta_{i}(A) \delta_{j}\left(G_{1}\right) \delta_{k}(B)+\sum_{i+j=n} \delta_{i}(A) \delta_{j}(B) \\
& -\sum_{i+j+k=n} \delta_{i}(A) \delta_{j}\left(G_{1}\right) \delta_{k}(B) \\
= & \sum_{i+j=n} \delta_{i}(A) \delta_{j}(B)
\end{aligned}
$$

for all $A, B \in \operatorname{Alg} \mathcal{L}$. This shows that $D=\left(\delta_{i}\right)_{i \in \mathbb{N}}$ is a higher derivation from $\operatorname{Alg} \mathcal{L}$ into itself if $G_{1} \vee G_{2}=I$.

Case 2. Suppose that $G_{1} \vee G_{2}<I$.

Let $G=G_{1} \vee G_{2}$. Since $G \in \mathcal{L} \cap \mathcal{L}^{\perp}$ and $\operatorname{Alg} \mathcal{L}(I-G) \subseteq \mathcal{L}^{\prime}$, then $(I-G)$ $\operatorname{Alg} \mathcal{L}(I-G)$ is a von Neumann algebra. The algebra $\operatorname{Alg} \mathcal{L}$ can be written as the direct sum

$$
\operatorname{Alg} \mathcal{L}=\operatorname{Alg}(G \mathcal{L} G) \oplus \operatorname{Alg}((I-G) \mathcal{L}(I-G)) .
$$

Since $G \in \mathcal{L} \cap \mathcal{L}^{\perp}$, by Lemma 2.2, we have $\delta_{n}(G)=0$ for all $n \geq 1$ and $\delta_{n}(I-G)=0$ for all $n \geq 1$. Hence

$$
\delta_{n}(G A G)=G \delta_{n}(A) G \quad \text { and } \quad \delta_{n}((I-G) A(I-G))=(I-G) \delta_{n}(A)(I-G)
$$

for every $A \in \operatorname{Alg} \mathcal{L}$. Therefore $D$ can be written as $D^{(1)} \oplus D^{(2)}$, where $D^{(1)}$ is a Jordan higher derivation from $\operatorname{Alg}(G \mathcal{L} G)$ into itself and $D^{(2)}$ is a Jordan higher derivation from $\operatorname{Alg}((I-G) \mathcal{L}(I-G))$ into itself. It is easy to show that $G_{1}(G \mathcal{L} G) \vee$ $G_{2}(G \mathcal{L} G)=G$. So it follows from Case 1 that $D^{(1)}$ is a higher derivation. Since every von Neumann algebra is a semiprime ring, $D^{(2)}$ is also a higher derivation by [6, Theorem 1.2]. Consequently, $D=\left(\delta_{i}\right)_{i \in \mathbb{N}}$ is a higher derivation from $\operatorname{Alg} \mathcal{L}$ into itself.

\section{High derivable linear mapping at some points}

Let $\mathcal{A}$ denote a unital Banach algebra and $D=\left(\delta_{i}\right)_{i \in \mathbb{N}}: \mathcal{A} \rightarrow \mathcal{A}$ be a family of linear mappings. We say that $D$ is a higher derivable linear mapping at $A$ if $\delta_{n}(x y)=$ $\sum_{i+j=n} \delta_{i}(x) \delta_{j}(y)$ for all $n \in N$ and $x, y \in \mathcal{A}$ with $x y=A$. In the following, 
we study the conditions under which higher derivations of $\mathcal{A}$ can be completely determined by the action on the zero-product elements.

LEMMA 3.1. Let $\mathcal{A}$ be a unital Banach algebra and $D=\left(\delta_{i}\right)_{i \in \mathbb{N}}$ be a higher derivable linear mapping at 0 from $\mathcal{A}$ into itself with $\delta_{n}(I)=0$ for all $n \geq 1$. Then for every idempotent $P \in \mathcal{A}$, every $A \in \mathcal{A}$ and each $n \in \mathbb{N}$ :

(a) $\delta_{n}(P A)=\sum_{i+j=n} \delta_{i}(P) \delta_{j}(A)$;

(b) $\delta_{n}(A P)=\sum_{i+j=n} \delta_{i}(A) \delta_{j}(P)$.

PROOF. (a) From

$$
\begin{aligned}
0=\delta_{n}(P(I-P) A) & =\sum_{i+j=n} \delta_{i}(P) \delta_{j}((I-P) A) \\
& =\sum_{i+j=n} \delta_{i}(P) \delta_{j}(A)-\sum_{i+j=n} \delta_{i}(P) \delta_{j}(P A)
\end{aligned}
$$

and

$$
\begin{aligned}
0=\delta_{n}((I-P) P A) & =\sum_{i+j=n} \delta_{i}(I-P) \delta_{j}(P A) \\
& =\sum_{i+j=n} \delta_{i}(I) \delta_{j}(P A)-\sum_{i+j=n} \delta_{i}(P) \delta_{j}(P A) \\
& =\delta_{n}(P A)-\sum_{i+j=n} \delta_{i}(P) \delta_{j}(P A)
\end{aligned}
$$

we obtain $\delta_{n}(P A)=\sum_{i+j=n} \delta_{i}(P) \delta_{j}(A)$ for every idempotent $P \in \mathcal{A}$, every $A \in \mathcal{A}$ and each $n \in \mathbb{N}$. With a proof similar to the proof of (a) we can show that (b) is also true.

Corollary 3.2. Let $\mathcal{A}$ and $D=\left(\delta_{i}\right)_{i \in \mathbb{N}}$ be as in Lemma 3.1 with $\delta_{n}(I)=0$ for all $n \geq 1$. Suppose that $\mathcal{B}$ is the subalgebra of $\mathcal{A}$ generated by all idempotents in $\mathcal{A}$. Then for any $T_{1}, T_{2} \in \mathcal{B}, A \in \mathcal{A}$ and each $n \in \mathbb{N}$ :

(a) $\delta_{n}\left(T_{1} A\right)=\sum_{i+j=n} \delta_{i}\left(T_{1}\right) \delta_{j}(A)$;

(b) $\delta_{n}\left(A T_{1}\right)=\sum_{i+j=n} \delta_{i}(A) \delta_{j}\left(T_{1}\right)$;

(c) $\delta_{n}\left(T_{1} T_{2}\right)=\sum_{i+j=n} \delta_{i}\left(T_{1}\right) \delta_{j}\left(T_{2}\right)$.

We call a left (right) ideal $\mathcal{I}$ of $\mathcal{A}$ a separating left (right) ideal of $\mathcal{M}$, if for every $m$ in $\mathcal{M}, m \mathcal{I}=0(\mathcal{I} m=0)$ implies $m=0$. An ideal $\mathcal{I}$ of $\mathcal{A}$ is called a separating ideal of $\mathcal{M}$ if $\mathcal{I}$ is a separating left ideal and a separating right ideal of $\mathcal{M}$.

Theorem 3.3. Let $\mathcal{A}$ and $D=\left(\delta_{i}\right)_{i \in \mathbb{N}}$ be as in Lemma 3.1 with $\delta_{n}(I)=0$ for all $n \geq 1$. If $\mathcal{I}$ is a separating left (right) ideal of $\mathcal{A}$ and $\mathcal{I}$ is contained in the subalgebra of $\mathcal{A}$ generated by all idempotents in $\mathcal{A}$, then $D$ is a higher derivation. 
Proof. Suppose that $\mathcal{I}$ is a separating left ideal of $\mathcal{A}$. When $n=1$, it is easy to show that $\delta_{1}$ is a derivation, that is, $\delta_{1}(A B)=\delta_{1}(A) B+A \delta_{1}(B)$ for all $A, B \in \mathcal{A}$. Now we assume that

$$
\delta_{m}(A B)=\sum_{i+j=m} \delta_{i}(A) \delta_{j}(B)
$$

for all $A, B \in \mathcal{A}$ and for all $1 \leq m<n$.

Let $A, B \in \mathcal{A}$. Then by Corollary 3.2, for $S \in \mathcal{I}$,

$$
\begin{aligned}
& \delta_{n}(A B S)=\sum_{i+j=n} \delta_{i}(A B) \delta_{j}(S)=\delta_{n}(A B) S+\sum_{i+j=n} \delta_{i}(A B) \delta_{j}(S), \\
& \delta_{n}(A B S)=\sum_{i+j=n} \delta_{i}(A) \delta_{j}(B S)=\sum_{i+j+k=n} \begin{array}{c}
i \geq 1 \\
\delta_{i}(A) \delta_{j}(B) \delta_{k}(S)
\end{array} \\
& =\sum_{i+j=n} \delta_{i}(A) \delta_{j}(B) S+\sum_{\substack{i+j+k=n \\
k \geq 1}} \delta_{i}(A) \delta_{j}(B) \delta_{k}(S) .
\end{aligned}
$$

So by the induction hypothesis, we obtain

$$
\left(\delta_{n}(A B)-\sum_{i+j=n} \delta_{i}(A) \delta_{j}(B)\right) S=0 .
$$

Since $\mathcal{I}$ is a separating left ideal of $\mathcal{A}$, it follows that

$$
\delta_{n}(A B)=\sum_{i+j=n} \delta_{i}(A) \delta_{j}(B) .
$$

So $D$ is a higher derivation.

Suppose that $\mathcal{I}$ is a separating right ideal of $\mathcal{A}$. We can similarly prove that $D$ is a higher derivation.

LEMMA 3.4. Let $\mathcal{L}$ be a CSL on $H$. If $D=\left(\delta_{i}\right)_{i \in \mathbb{N}}$ is a higher derivable linear mapping at 0 from $\operatorname{Alg} \mathcal{L}$ into itself and $\delta_{n}(I)=0$ for all $n \geq 1$, then for any $P \in \mathcal{L}$ and $n \geq 1$ :

(a) $\delta_{n}(P)=P \delta_{n}(P)(I-P)$;

(b) $\delta_{n}(I-P)=P \delta_{n}(I-P)(I-P)$.

PRoOF. When $n=1$, we can easily obtain $\delta_{1}(P)=P \delta_{1}(P)(I-P)$. Now we assume that

$$
\delta_{m}(P)=P \delta_{m}(P)(I-P)
$$

for all $1 \leq m<n$. By Lemma 3.1, it follows that

$$
\begin{aligned}
\delta_{n}(P) & =\sum_{i+j=n} \delta_{i}(P) \delta_{j}(P) \\
& =P \delta_{n}(P)+\delta_{n}(P) P+\sum_{\substack{i+j=n \\
i, j \geq 1}} \delta_{i}(P) \delta_{j}(P) \\
& =P \delta_{n}(P)+\delta_{n}(P) P .
\end{aligned}
$$


This means that

$$
\delta_{n}(P)=P \delta_{n}(P)(I-P)
$$

and

$$
\delta_{n}(I-P)=-\delta_{n}(P)=-P \delta_{n}(P)(I-P)=P \delta_{n}(I-P)(I-P) \quad \text { for all } n \geq 1 .
$$

This concludes the proof.

THEOREM 3.5. Let $\mathcal{L}$ be a CSL on $H$. If $D=\left(\delta_{i}\right)_{i \in \mathbb{N}}$ is a bounded higher derivable linear mapping at 0 from $\mathrm{Alg} \mathcal{L}$ into itself and $\delta_{n}(I)=0$ for all $n \geq 1$, then $D=$ $\left(\delta_{i}\right)_{i \in \mathbb{N}}$ is a higher derivation.

PROOF. We divide the proof into two cases.

Case 1. Suppose that $G_{1} \vee G_{2}=I$.

The proof is similar to the proof of Case 1 in Theorem 2.5.

Case 2. Suppose that $G_{1} \vee G_{2}<I$.

Let $G=G_{1} \vee G_{2}$. The algebra $\operatorname{Alg} \mathcal{L}$ can be written as the direct sum

$$
\operatorname{Alg} \mathcal{L}=\operatorname{Alg}(G \mathcal{L} G) \oplus \operatorname{Alg}((I-G) \mathcal{L}(I-G)) .
$$

Since $G \in \mathcal{L} \cap \mathcal{L}^{\perp}$, by Lemma 3.4, we have $\delta_{n}(G)=0$ and $\delta_{n}(I-G)=0$ for all $n \geq 1$. Hence

$$
\delta_{n}(G A G)=G \delta_{n}(A) G \quad \text { and } \quad \delta_{n}((I-G) A(I-G))=(I-G) \delta_{n}(A)(I-G)
$$

for every $A \in \operatorname{Alg} \mathcal{L}$. Therefore $D$ can be written as $D^{(1)} \oplus D^{(2)}$, where $D^{(1)}$ is a bounded higher derivable linear mapping at 0 from $\operatorname{Alg}(G \mathcal{L} G)$ into itself and $D^{(2)}$ is a bounded higher derivable linear mapping at 0 from $\operatorname{Alg}((I-G) \mathcal{L}(I-G))$ into itself. It is easy to show that $G_{1}(G \mathcal{L} G) \vee G_{2}(G \mathcal{L} G)=G$. So it follows from Case 1 that $D^{(1)}$ is a higher derivation. Since $(I-G) \operatorname{Alg} \mathcal{L}(I-G)$ is a von Neumann algebra and $D^{(2)}$ is continuous, $D^{(2)}$ is a higher derivation by Corollary 3.2. Consequently, $D=\left(\delta_{i}\right)_{i \in \mathbb{N}}$ is a higher derivation.

In the following, we study the higher derivable linear mapping at unit-product elements on Banach algebras.

LemMa 3.6. Let $\mathcal{A}$ be a unital Banach algebra and $D=\left(\delta_{i}\right)_{i \in \mathbb{N}}: \mathcal{A} \rightarrow \mathcal{A}$ be a family of linear mappings such that

$$
\sum_{i+j=n} \delta_{i}(A) \delta_{j}\left(A^{-1}\right)=0 \quad \text { for all } n \geq 1
$$

and for every invertible element $A \in \mathcal{A}$. Then $D=\left(\delta_{i}\right)_{i \in \mathbb{N}}$ is a Jordan higher derivation. 
Proof. It is easy to show that $\delta_{n}(I)=0$ for all $n \geq 1$. When $n=1, \delta_{1}(A) A^{-1}+$ $A \delta_{1}\left(A^{-1}\right)=0$ for every invertible element $A \in \mathcal{A}$. Then with the similar proof of [12, Theorem 2.2], we can show that $\delta_{1}$ is a Jordan derivation, that is, $\delta_{1}\left(B^{2}\right)=$ $\delta_{1}(B) B+B \delta_{1}(B)$ for every $B$ in $\mathcal{A}$. Now we assume that

$$
\delta_{m}\left(B^{2}\right)=\sum_{i+j=m} \delta_{i}(B) \delta_{j}(B)
$$

for all $1 \leq m<n$ and every $B$ in $\mathcal{A}$. For any $T \in \mathcal{A}$, let $n$ be a positive integer such that $n \geq\|T\|+1$ and $A=n I+T$. We know that $A$ and $I-A$ are invertible in $\mathcal{A}$. So

$$
\begin{aligned}
\delta_{n}(A)= & -\sum_{\substack{i+j=n \\
j \geq 1}} \delta_{i}(A) \delta_{j}\left(A^{-1}\right) A \\
= & -\sum_{\substack{i+j=n \\
j \geq 1}} \delta_{i}(A) \delta_{j}\left(A^{-1}(I-A)^{2}\right) A+\sum_{\substack{i+j=n \\
j \geq 1}} \delta_{i}(A) \delta_{j}(A) A \\
= & \sum_{\substack{i+e+f=n \\
f \geq 1}} \delta_{i}(A) \delta_{e}\left(A^{-1}(I-A)^{2}\right) \delta_{f}\left((I-A)^{-2} A\right)(I-A)^{2} \\
& +\sum_{\substack{i+j=n \\
j \geq 1}} \delta_{i}(A) \delta_{j}(A) A \\
= & \sum_{\substack{i+e+f=n \\
f \geq 1}} \delta_{i}(A) \delta_{e}\left(A^{-1}+A-2 I\right) \delta_{f}\left((I-A)^{-2}\right)(I-A)^{2} \\
& -\sum_{\substack{i+e+f=n \\
f \geq 1}} \delta_{i}(A) \delta_{e}\left(A^{-1}+A-2 I\right) \delta_{f}\left((I-A)^{-1}\right)(I-A)^{2} \\
& +\sum_{\substack{i+j=n \\
j \geq 1}} \delta_{i}(A) \delta_{j}(A) A .
\end{aligned}
$$

By a simple calculation and the induction hypothesis,

$$
\begin{aligned}
& \sum_{\substack{i+e+f=n \\
f \geq 1}} \delta_{i}(A) \delta_{e}\left(A^{-1}+A-2 I\right) \delta_{f}\left((I-A)^{-2}\right)(I-A)^{2} \\
& \quad=\sum_{\substack{i+j=n \\
j \geq 1}} \delta_{i}\left((I-A)^{2}\right) \delta_{j}\left((I-A)^{-2}\right)(I-A)^{2} \\
& \quad=-\delta_{n}\left((I-A)^{2}\right)=\delta_{n}(2 A)-\delta_{n}\left(A^{2}\right)
\end{aligned}
$$


and

$$
\begin{aligned}
& -\sum_{\substack{i+e+f=n \\
f \geq 1}} \delta_{i}(A) \delta_{e}\left(A^{-1}+A-2 I\right) \delta_{f}\left((I-A)^{-1}\right)(I-A)^{2} \\
& \quad=-\sum_{\substack{i+j=n \\
j \geq 1}} \delta_{i}(I-A) \delta_{j}\left((I-A)^{-1}\right)(I-A)^{2}-\sum_{\substack{i+j=n \\
j \geq 1}} \delta_{i}(A) \delta_{j}(I-A)(I-A) \\
& \quad=\delta_{n}(I-A)(I-A)+\sum_{\substack{i+j=n \\
j \geq 1}} \delta_{i}(A) \delta_{j}(A)(I-A) \\
& \quad=-\delta_{n}(A)+\delta_{n}(A) A+\sum_{\substack{i+j=n \\
j \geq 1}} \delta_{i}(A) \delta_{j}(A)-\sum_{\substack{i+j=n \\
j \geq 1}} \delta_{i}(A) \delta_{j}(A) A .
\end{aligned}
$$

So we have

$$
\delta_{n}(A)=\delta_{n}(A)-\delta_{n}\left(A^{2}\right)+\delta_{n}(A) A+\sum_{\substack{i+j=n \\ j \geq 1}} \delta_{i}(A) \delta_{j}(A) .
$$

Hence we obtain, for every invertible element $A \in \mathcal{A}$,

$$
\delta_{n}\left(A^{2}\right)=\sum_{i+j=n} \delta_{i}(A) \delta_{j}(A) .
$$

Since $\delta_{n}(I)=0$ for all $n \geq 1$, we have, for every $T \in \mathcal{A}$,

$$
\delta_{n}\left(T^{2}\right)=\sum_{i+j=n} \delta_{i}(T) \delta_{j}(T) .
$$

This concludes the proof.

THEOREM 3.7. Let $\mathcal{A}$ be a unital Banach algebra and $D=\left(\delta_{i}\right)_{i \in \mathbb{N}}$ be a family of linear mappings from $\mathcal{A}$ into itself. Then $D=\left(\delta_{i}\right)_{i \in \mathbb{N}}$ is a higher derivable linear mapping at $I$ if and only if $D=\left(\delta_{i}\right)_{i \in \mathbb{N}}$ is a Jordan higher derivation.

Proof. Let $D=\left(\delta_{i}\right)_{i \in \mathbb{N}}$ be a higher derivable linear mapping at $I$. Then, by Lemma 3.6, $D=\left(\delta_{i}\right)_{i \in \mathbb{N}}$ is a Jordan higher derivation. Conversely, if $D=\left(\delta_{i}\right)_{i \in \mathbb{N}}$ is a Jordan higher derivation, then $\delta_{1}$ is a Jordan derivation. So $\delta_{1}$ is a derivable linear mapping at $I$. Now we assume that $\sum_{i+j=m} \delta_{i}(A) \delta_{j}(B)=0$ for all $A, B \in \mathcal{A}$ with $A B=I$ and $1 \leq m<n$. Thus for all $A, B \in \mathcal{A}$ with $A B=I$,

$$
\begin{aligned}
\delta_{n}(B) & =\delta_{n}(B A B)=\sum_{i+j+k=n} \delta_{i}(B) \delta_{j}(A) \delta_{k}(B) \\
& =B \sum_{i+j=n} \delta_{i}(A) \delta_{j}(B)+\sum_{\substack{i+j+k=n \\
i \geq 1}} \delta_{i}(B) \delta_{j}(A) \delta_{k}(B) \\
& =B \sum_{i+j=n} \delta_{i}(A) \delta_{j}(B)+\delta_{n}(B) .
\end{aligned}
$$


So

$$
B \sum_{i+j=n} \delta_{i}(A) \delta_{j}(B)=0 \text { for all } n \geq 1
$$

Hence

$$
\sum_{i+j=n} \delta_{i}(A) \delta_{j}(B)=A B \sum_{i+j=n} \delta_{i}(A) \delta_{j}(B)=0
$$

for all $n \geq 1$. This means that $D=\left(\delta_{i}\right)_{i \in \mathbb{N}}$ is a higher derivable linear mapping at $I$.

In Section 1, we have proved that if $D=\left(\delta_{i}\right)_{i \in \mathbb{N}}$ is a Jordan higher derivation on a CSL algebra, then $D=\left(\delta_{i}\right)_{i \in \mathbb{N}}$ is a higher derivation. So by Theorem 3.7, we have the following corollary.

COROLlary 3.8. Let $\mathcal{L}$ be a CSL on $H$. If $D=\left(\delta_{i}\right)_{i \in \mathbb{N}}$ is a higher derivable linear mapping at I from $\mathrm{Alg} \mathcal{L}$ into itself, then $D=\left(\delta_{i}\right)_{i \in \mathbb{N}}$ is a higher derivation.

\section{References}

[1] J. Alaminos, J. Extremera, A. Villena and M. Brešar, 'Characterizing homomorphisms and derivations on $C^{*}$-algebras', Proc. Roy. Soc. Edinburgh Sect. A 137 (2007), 1-7.

[2] M. Brešar, 'Jordan derivations on semiprime rings', Proc. Amer. Math. Soc. 104 (1988), 1003-1006.

[3] M. Chebotar, W. Ke and P. Lee, 'Maps characterized by action on zero products', Pacific J. Math. 216 (2004), 217-228.

[4] R. Crist, 'Local derivations on operator algebras', J. Funct. Anal. 135 (1996), 76-92.

[5] K. Davidson, Nest Algebras, Pitman Research Notes in Mathematics Series, 191 (Longman Scientific, Harlow, 1988).

[6] M. Ferrero and C. Haetinger, 'Higher derivations and a theorem by Herstein', Quaest. Math. 25 (2002), 249-257.

[7] I. Herstein, 'Jordan derivations of prime rings', Proc. Amer. Math. Soc. 8 (1957), 1104-1110.

[8] W. Jing, S. Lu and P. Li, 'Characterisations of derivations on some operator algebras', Bull. Aust. Math. Soc. 66 (2002), 227-232.

[9] B. Johnson, 'Local derivations on $C^{*}$-algebras are derivations', Trans. Amer. Math. Soc. 353 (2001), 313-325.

[10] R. Kadison, 'Local derivations', J. Algebra 130 (1990), 494-509.

[11] J. Li and Z. Pan, 'Annihilator-preserving maps, multipliers, and derivations', Linear Algebra Appl. 432 (2010), 5-13.

[12] F. Lu, 'Characterizations of derivations and Jordan derivations on Banach algebras', Linear Algebra Appl. 430 (2009), 2233-2239.

[13] F. Lu, 'The Jordan structure of CSL algebras', Studia Math. 190 (2009), 283-299.

[14] A. Nakajima, 'On generalized higher derivations', Turkish J. Math. 24 (2000), 295-311.

[15] Z. Xiao and F. Wei, 'Jordan higher derivations on triangular algebras', Linear Algebra Appl. 432 (2010), 2615-2622.

JIANKUI LI, Department of Mathematics, East China University of Science and Technology, Shanghai 200237, PR China e-mail: jiankuili@yahoo.com

JIANBIN GUO, Department of Mathematics, East China University of Science and Technology, Shanghai 200237, PR China e-mail: jianbin-guo@163.com 